University of Nebraska - Lincoln DigitalCommons@University of Nebraska - Lincoln

1992

\title{
On spurious asymptotic numerical solutions of explicit Runge Kutta methods
}

D. F. Griffiths

University of Dundee

P. K. Sweby

University of Reading

Helen C. Yee

NASA Ames Research Center, yee@nas.nasa.gov

Follow this and additional works at: http://digitalcommons.unl.edu/nasapub

Griffiths, D. F.; Sweby, P. K.; and Yee, Helen C., "On spurious asymptotic numerical solutions of explicit Runge Kutta methods" (1992). NASA Publications. 244.

http://digitalcommons.unl.edu/nasapub/244

This Article is brought to you for free and open access by the National Aeronautics and Space Administration at DigitalCommons@University of Nebraska - Lincoln. It has been accepted for inclusion in NASA Publications by an authorized administrator of DigitalCommons@University of Nebraska - Lincoln. 


\title{
On spurious asymptotic numerical solutions of explicit Runge-Kutta methods $\dagger$
}

\author{
D. F. GRIFFTHS $\ddagger$ \\ Department of Mathematics, University of Dundee, Scotland \\ P. K. SwEBy $\ddagger$ \\ Department of Mathematics, University of Reading, England \\ AND \\ H. C. YeEף \\ NASA Ames Research Center, Moffett Field, CA 94035, USA \\ [Received 28 July 1990 and in revised form 3 October 1991]
}

\begin{abstract}
The bifurcation diagram associated with the logistic equation $v^{n+1}=a v^{n}\left(1-v^{n}\right)$ is by now well known, as is its equivalence to solving the ordinary differential equation (ODE) $u^{\prime}=\alpha u(1-u)$ by the explicit Euler difference scheme. It has also been noted by Iserles that other popular difference schemes may not only exhibit period doubling and chaotic phenomena but also possess spurious fixed points. We investigate, both analytically and computationally, Runge-Kutta schemes applied to the equation $u^{\prime}=f(u)$, for $f(u)=\alpha u(1-u)$ and $f(u)=$ $a u(1-u)(b-u)$, contrasting their behaviour with the explicit Euler scheme. We determine and provide a local analysis of bifurcations to spurious fixed points and periodic orbits. In particular we show that these may appear below the linearised stability limit of the scheme, and may consequently lead to erroneous computational results.
\end{abstract}

\section{Introduction}

It is now well established that numerical schemes for solving ordinary differential equations (ODEs) may exhibit period-doubling and chaotic behaviour when used with time steps above their linearised stability limit. The most well known example of this is the explicit Euler difference scheme applied to the ODE

$$
u^{\prime}=\alpha u(1-u) \text {. }
$$

For this equation the scheme becomes

$$
u^{n+1}=u^{n}+\alpha \Delta t u^{n}\left(1-u^{n}\right)
$$

where $\Delta t$ is the timestep being used, and its associated bifurcation diagram is shown in Fig. 1. The bifurcation diagram is a plot of $u^{n}$ against $r=\alpha \Delta t$ for two hundred iterations, after the first 600 iterates have been taken to allow any

† Major part of the material was published as an internal report-NASA Technical Memorandum 102919, April 1990, also as University of Reading Numerical Analysis Report 3/90, March 1990.

¥ This work was performed whilst a visiting scientist at NASA Ames Research Center, Moffett Field. CA 94035 USA.

ף Staff Scientist, Fluid Dynamics Division. 
transient phase to be completed. As can be seen, for $r<2$ (the linearised absolute stability limit at the stationary point $u=1$ ) all the successive iterates take the value 1 , the stable equilibrium of the differential equation. Above this value of $r$ the iterates alternate between two values whilst for even larger values of $r$ the iterates cycle among four distinct values and so on. This phenomenon is known as period doubling, and as $r$ increases, degenerates into chaotic behaviour where no finite set of distinct values can be discerned. Finally, at $r=3$ the numerical scheme breaks down as its solutions diverge to infinity. Notice however how the period-doubling behaviour is interrupted by solutions of lower periods, a feature of most bifurcation diagrams of simple discrete maps [16]. The numbers labelling the branches of the bifurcation diagram in Fig. 1 indicate their period (up to period 8), in addition the subscript $E$ on the period one label indicates that it is an essential fixed point-that is, a fixed point of the ODE (1.1). We shall see later that other, spurious, fixed points may be produced by some numerical schemes.

This type of period doubling behaviour is well known, the above example being equivalent, after a linear transformation, to the logistic equation of population

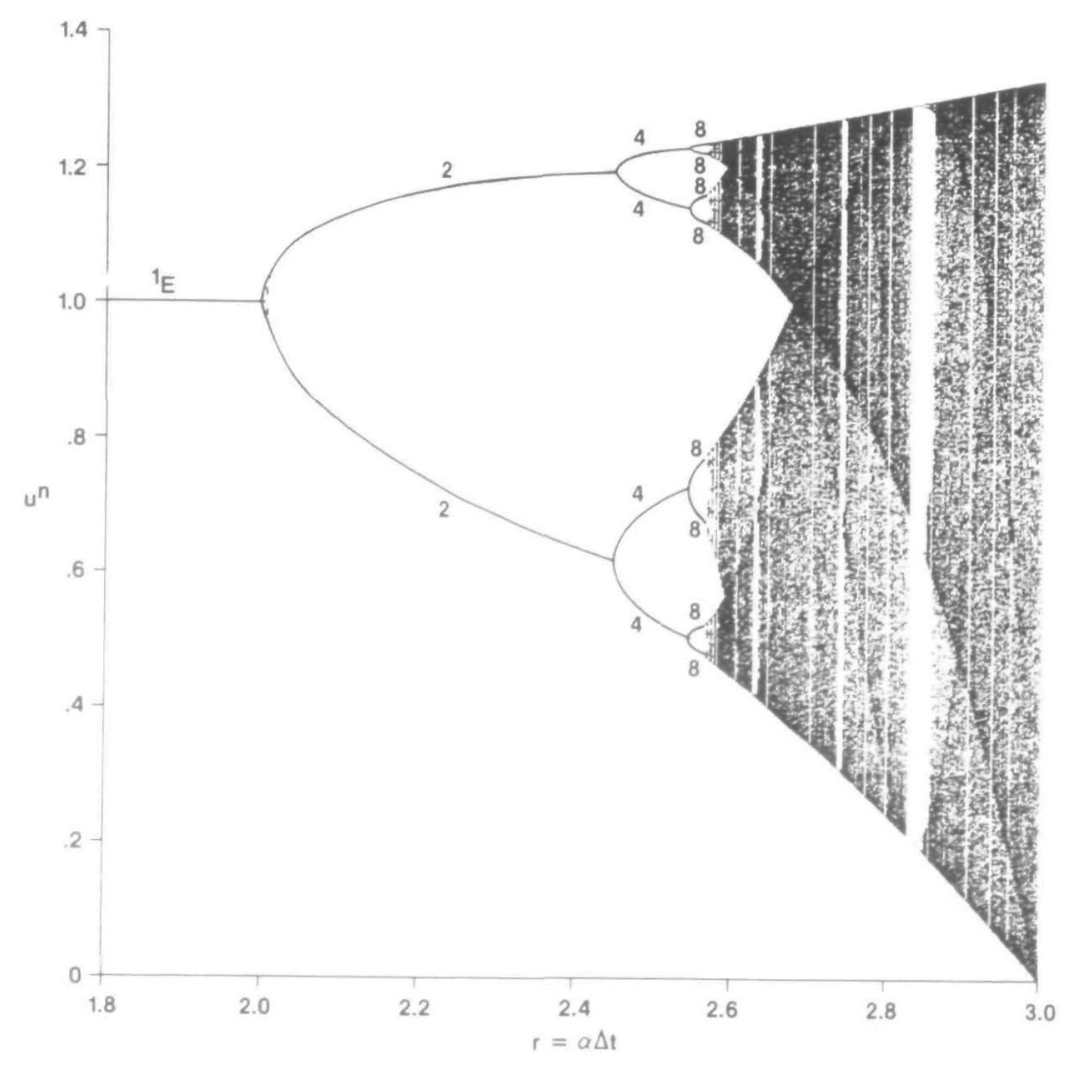


dynamics [14]

$$
v^{n+1}=a v^{n}\left(1-v^{n}\right),
$$

which is known for its chaotic behaviour [9].

It is to be noted that once the explicit Euler scheme (which is both a linear multi-step scheme and a first order, one-stage Runge-Kutta method) exceeds its linearised stability limit it announces the fact by its period 2 behaviour where the solution oscillates between two values. This is because the fixed points of linear multistep methods, the explicit Euler scheme being a simple example, coincide with those of the differential equation (Iserles [5]). This means that if the iterates converge for fixed $\Delta t$ as $n \rightarrow \infty$, i.e. tend to a period 1 solution, then this is a solution of the differential equation. However, Iserles [5] has shown that this need not be the case for Runge-Kutta schemes and, as we shall show later, these schemes can produce spurious solutions which, despite being non-oscillatory (period 1), are not solutions to the differential equation. In the terminology of $[4,5]$ such methods are not regular. We investigate this phenomenon for several popular Runge-Kutta schemes and observe that such spurious solutions may, in some circumstances, be obtained for values of $r$ below the linearised stability limit (this observation is not new, see for example [5,11] and also [1] where spurious invariant curves were found for $\Delta t$ sufficiently small). These solutions are invariably of large amplitude (proportional to a negative power of $\Delta t$ ) which would be an indicator of their spurious nature. Their presence may nevertheless affect computations in that they generally restrict the range of initial data that are attracted to the true fixed point. We also note that for these schemes we must sometimes greatly exceed the linearised stability limit before oscillatory behaviour hints towards a spurious solution even though such a solution has been present since the limit was reached. This situation does not necessarily require that the time step be unreasonably large since, at the linearised stability limit, $\Delta t=$ $O(1 / \alpha)$ and will thus be small for fast reactions $(\alpha \gg 1)$. Such time steps are of the same order of magnitude as those that would be generated asymptotically by variable time-stepping methods with error control $[3,12]$. However, these more sophisticated methods are far more robust and we outline one reason why their use should preclude spurious fixed points at the end of Section 3.

The implications of the behaviour detailed above ranges far beyond pure ODEs. For example, iterative schemes for many steady state calculations for partial differential equations (PDEs) are constructed from ODE methods, often of Runge-Kutta type, to 'time' march the solution. Could therefore the behaviour observed in this paper explain instances of non-convergence where the iterates appear to oscillate between more than one steady state, or where the residual will decrease only so far before reaching a plateau? We believe that this could well be the case, though the mechanisms involved are far more complicated than those studied here.

This then leads us to ask whether computed steady states, which show no oscillatory behaviour, are approximations to true solutions to the differential equations? We indicate how this question may be addressed in Section 3 but our 
main objective is a detailed examination of bifurcation diagrams, the tracking of the lower periodic fixed points of the schemes, and their determination by analytic means where possible.

The consequences of this kind of dynamical behaviour for finite difference methods for practical PDEs in computational fluid dynamics is discussed in Yee [19] and Yee $e t$ al. [20]. A general account of the theory of asymptotic states of numerical methods applied to initial value problems may be found in Iserles et al. [6]. Some results pertaining to implicit Runge-Kutta methods and their unique solvability in the neighbourhood of asymptotically stable equilibria, independent of the grid size, are given in [13].

In the next section we describe the specific Runge-Kutta methods used in our experiments and determine their fixed points both for the equation (1.1) and for

$$
u^{\prime}=\alpha u(1-u)(b-u)
$$

for constant $b$ lying in the range $0<b<1$. Then, in Section 3 , we use local perturbation theory to show how certain spurious fixed points bifurcate from the true fixed point at the linearised absolute stability limit of the methods. Since not all fixed points bifurcate in this way, we also discuss how other branches may be determined asymptotically as $\Delta t \rightarrow \infty$. Higher order periodic orbits of the numerical schemes are then examined in Section 4.

\section{Preliminary study for specific methods}

We shall consider Runge-Kutta methods applied first to the general scalar ordinary differential equation

$$
u^{\prime}=f(u)
$$

where $f(u)$ is a non-linear function of the variable $u$. In our examples, the form of $f$ will be given as in (1.1) and (1.4).

This equation has fixed points $u^{*}$ (also known as equilibrium points, critical points or steady-state solutions) when

$$
f\left(u^{*}\right)=0 \text {, }
$$

i.e. when the equation (2.1) is in equilibrium. When $f^{\prime}\left(u^{*}\right)<0$ the fixed point is stable (neighbouring solutions are attracted to it exponentially) whereas it is unstable if $f^{\prime}\left(u^{*}\right)>0$ (neighbouring solutions are repelled). The case $f^{\prime}\left(u^{*}\right)=0$ is degenerate and stability of the fixed point cannot be established by linearising the differential equation.

It is easily established that equation (1.1) has a stable fixed point $u^{*}=1$ and an unstable fixed point $u^{*}=0$ while (1.4) is stable at $u^{*}=b$ and has two unstable fixed points $u^{*}=0,1$.

In this section we shall discuss the following five explicit Runge-Kutta schemes (the theory of the next section being valid more generally):

Explicit Euler, a 1-stage, first order method (This is also a linear multistep method):

$$
u^{n+1}=u^{n}+\Delta t f\left(u^{n}\right)
$$


Modified Euler, a 2-stage, second order method given by:

$$
u^{n+1}=u^{n}+\Delta t f\left(u^{n}+\frac{1}{2} \Delta t f\left(u^{n}\right)\right),
$$

Improved Euler, another 2-stage, second order method:

$$
u^{n+1}=u^{n}+\frac{1}{2} \Delta t\left[f\left(u^{n}\right)+f\left(u^{n}+\Delta t f\left(u^{n}\right)\right)\right] .
$$

Heun, a 3-stage, third order method:

$$
\begin{aligned}
u^{n+1} & =u^{n}+\frac{\Delta t}{4}\left(k_{1}+3 k_{3}\right), \\
k_{1} & =f\left(u^{n}\right), \\
k_{2} & =f\left(u^{n}+\frac{1}{3} \Delta t k_{1}\right), \\
k_{3} & =f\left(u^{n}+\frac{2}{3} \Delta t k_{2}\right) .
\end{aligned}
$$

Fourth order Runge-Kutta, the classical 4-stage, fourth order method:

$$
\begin{aligned}
u^{n+1} & =u^{n}+\frac{\Delta t}{6}\left(k_{1}+2 k_{2}+2 k_{3}+k_{4}\right), \\
k_{1} & =f\left(u^{n}\right), \\
k_{2} & =f\left(u^{n}+\frac{1}{2} \Delta t k_{1}\right), \\
k_{3} & =f\left(u^{n}+\frac{1}{2} \Delta t k_{2}\right), \\
k_{4} & =f\left(u^{n}+\Delta t k_{3}\right) .
\end{aligned}
$$

Each of these methods may be written in the general form

$$
u^{n+1}=u^{n}+\Delta t \Phi\left(u^{n}, \Delta t\right)
$$

and their fixed points are then the values $u_{\Delta}^{*}$ such that $u^{n}=u_{\Delta}^{*}$ for all $n$ and consequently satisfy

$$
\Phi\left(u_{\Delta}^{*}, \Delta t\right)=0 .
$$

The subscript $\Delta$ is used to indicate that the fixed points may now depend on the additional parameter $\Delta t$. Their stability may be investigated by linearisation in a similar manner to that used for the differential equation. First we perturb the fixed point by writing $u^{n}=u_{\Delta}^{*}+\delta^{n}$. Substituting this into (2.8) and neglecting second and higher order terms in $\delta^{n}$ leads to the linearised difference equation

$$
\delta^{n+1}=\delta^{n}\left[1+\Delta t \Phi_{u}\left(u_{\Delta}^{*}, \Delta t\right)\right]
$$

for $\delta^{n}$. The expression

$$
R(u, \Delta t)=1+\Delta t \Phi_{u}(u, \Delta t)
$$

is known as the linearised stability function of the method (2.8) at the point $(u, \Delta t)$. For stability we require that the perturbations decay with $n$ and this will be the case if

$$
\left|R\left(u_{\Delta}^{*}, \Delta t\right)\right|<1 .
$$


Because the parameter $\Delta t$ appears in these conditions (maybe not in a linear fashion) we shall generally have stability only for a certain range of $\Delta t$ values and, in the case $u_{\Delta}^{*}=u^{*}$ (a true fixed point of (2.1)), leads to the absolute stability limits and absolute stability intervals for the method [7]. For the problems (1.1) and (1.4) the parameter $\alpha$ acts as a scaling of the timestep $\Delta t$ and we shall therefore define

$$
r=\alpha \Delta t
$$

for notational convenience. We shall assume throughout that $r>0$.

Many of the stable fixed points for the various schemes applied to equations (1.1) and (1.4) may be determined (with the assistance of algebraic manipulation packages such as MAPLE [8] and DERIVE [2]) and the results we have been able to obtain in closed form are listed in Tables 2.1 and 2.2. Some entries are marked with an asterisk to indicate where stable fixed points are known to exist but no closed analytic form has yet been found. The stable ranges quoted in these cases have been determined from numerical computations.

To better understand the results of these Tables, we observe from (2.3)-(2.7) that $u_{\Delta}^{*}=u^{*}$ when $u^{*}$ satisfies (2.2): the true fixed points of the ODE are also fixed points of the Runge-Kutta methods. However, when $f$ is a polynomial of degree $m$ in $u$ and the Runge-Kutta method has $s$ stages, equation (2.9) represents a polynomial of degree $m^{s}$ in $u_{\Delta}^{*}$ (with coefficients depending on $\Delta t$ ). It is therefore possible for the number $\left(m^{s}-m\right)$ of spurious solutions, that is, roots of (2.9) that do not satisfy (2.2), to be large even for moderate values of $m$ and $s$. This is illustrated in Table 2.3 although not all the solutions counted will be

TABLE 2.1

Fixed points of Runge-Kutta methods for $u^{\prime}=u(1-u)$

\begin{tabular}{lcl}
\hline \multicolumn{1}{c}{ Scheme } & Fixed points & \multicolumn{1}{c}{ Stable range } \\
\hline Explicit Euler & 1 & $0<r<2$ \\
Modified Euler & 1 & $0<r<2$ \\
& $1+2 / r$ & $0<r<-1+\sqrt{5} \approx 1.236$ (Iserles [5]) \\
& $2 / r$ & $2<r<1+\sqrt{5} \approx 3.236$ (Iserles [5]) \\
Improved Euler & 1 & $0<r<2$ \\
& $\frac{\left[2+r \pm \sqrt{r^{2}-4}\right]}{2 r}$ & $2<r<\sqrt{8} \approx 2.828$ \\
Heun & 1 & $0<r<1+(\sqrt{17}+4)^{1 / 3}-(\sqrt{17}+4)^{-1 / 3} \approx 2.513$ \\
& $*$ & $4.9137<r<4.9552$ \\
& $*$ & $6.4799<r<6.4853$ \\
$R-K 4$ & $*$ & $6.74405<r<6.74575$ \\
& 1 & $0<r<\frac{4}{3}+\left(\frac{12}{77}+\frac{4}{3} \sqrt{29}\right)^{1 / 3}$ \\
& $*$ & $2.785<r<3.4156$ \\
& $*$ & $2 \cdot 746<r<3.456$ \\
\hline
\end{tabular}


TABLE 2.2

Fixed points of Runge-Kutta methods for $u^{\prime}=u(1-u)(b-u)$ in the symmetric case $b=\frac{1}{2}$

\begin{tabular}{|c|c|c|}
\hline Scheme & Fixed points & Stable range \\
\hline Explicit Euler & $\frac{1}{2}$ & $0<r<8$ \\
\hline \multirow[t]{4}{*}{ Modified Euler } & $\frac{1}{2}$ & $0<r<8$ \\
\hline & $\frac{1}{4}[1+\sqrt{1-32 / r}]$ & $32<r<32.014067$ \\
\hline & $\frac{1}{2}[1 \pm \sqrt{1-8 / r}]$ & $8<r<4(1+\sqrt{3}) \approx 10.928$ \\
\hline & $\frac{1}{d}[3-\sqrt{1-32 / r}]$ & $32<r<32.014067$ \\
\hline \multirow[t]{4}{*}{ Improved Euler } & $\frac{1}{2}$ & $0<r<8$ \\
\hline & $\frac{1 \pm \sqrt{1-8 / r}}{2}$ & $8<r<12$ \\
\hline & $\frac{1}{2}-\frac{\sqrt{1-12 / r}}{4} \pm \frac{\sqrt{1+4 / r}}{4}$ & $12<r<4(1+\sqrt{6}) \propto 13.798$ \\
\hline & $\frac{1}{2}+\frac{\sqrt{1-12 / r}}{4} \pm \frac{\sqrt{1+4 / r}}{4}$ & $12<r<4(1+\sqrt{6})=13.798$ \\
\hline Heun & $\frac{1}{2}$ & $\begin{aligned} 0<r<4 & \left(1+(\sqrt{17}+4)^{1 / 3}\right. \\
& \left.-(\sqrt{17}+4)^{-1 / 3}\right) \approx 10.051\end{aligned}$ \\
\hline$R-K 4$ & $\frac{1}{2}$ & $\begin{aligned} 0<r<\frac{16}{3} & +4\left(\frac{172}{27}+\frac{4}{3} \sqrt{29}\right)^{1 / 3} \\
& +4\left(\frac{172}{27}-\frac{4}{3} \sqrt{2} 9\right)^{1 / 3} \approx 11 \cdot 14\end{aligned}$ \\
\hline
\end{tabular}

anywhere stable, or even real valued. The existence of these spurious solutions and related issues will be discussed towards the end of the next section.

It is clearly not possible to detail all of these solutions and therefore only a representative sample has been given in Tables 2.1 and 2.2. In Table 2.1 we have listed all the stable fixed points except for those of $R-K 4$ where only the spurious fixed points occurring for the smallest values of $r$ are mentioned. On the other hand, in Table 2.2, only those fixed points that have been obtained in closed form are quoted.

Figures 2 and 3 show, respectively, the bifurcation diagrams for the differential equations (1.1) and (1.4), solved by each of the methods (2.4)-(2.7). Again the numeric labelling of the branches denotes their period, although some labels for period 4 and 8 are omitted due to the size of the figures. The subscript $E$ on period one branches indicates an essential fixed point of the differential equation

TABLE 2.3

The number of spurious fixed points of methods (2.3)-(2.7) for $u^{\prime}=u(1-u)$ and $u^{\prime}=u(1-u)\left(\frac{1}{2}-u\right)$

\begin{tabular}{cccccc}
\hline$f(u)$ & $(2 \cdot 3)$ & $(2 \cdot 4)$ & $(2 \cdot 5)$ & $(2 \cdot 6)$ & $(2 \cdot 7)$ \\
\hline$u(1-u)$ & 0 & 2 & 2 & 6 & 14 \\
$u\left(\frac{1}{2}-u\right)(1-u)$ & 0 & 6 & 6 & 24 & 78 \\
\hline
\end{tabular}



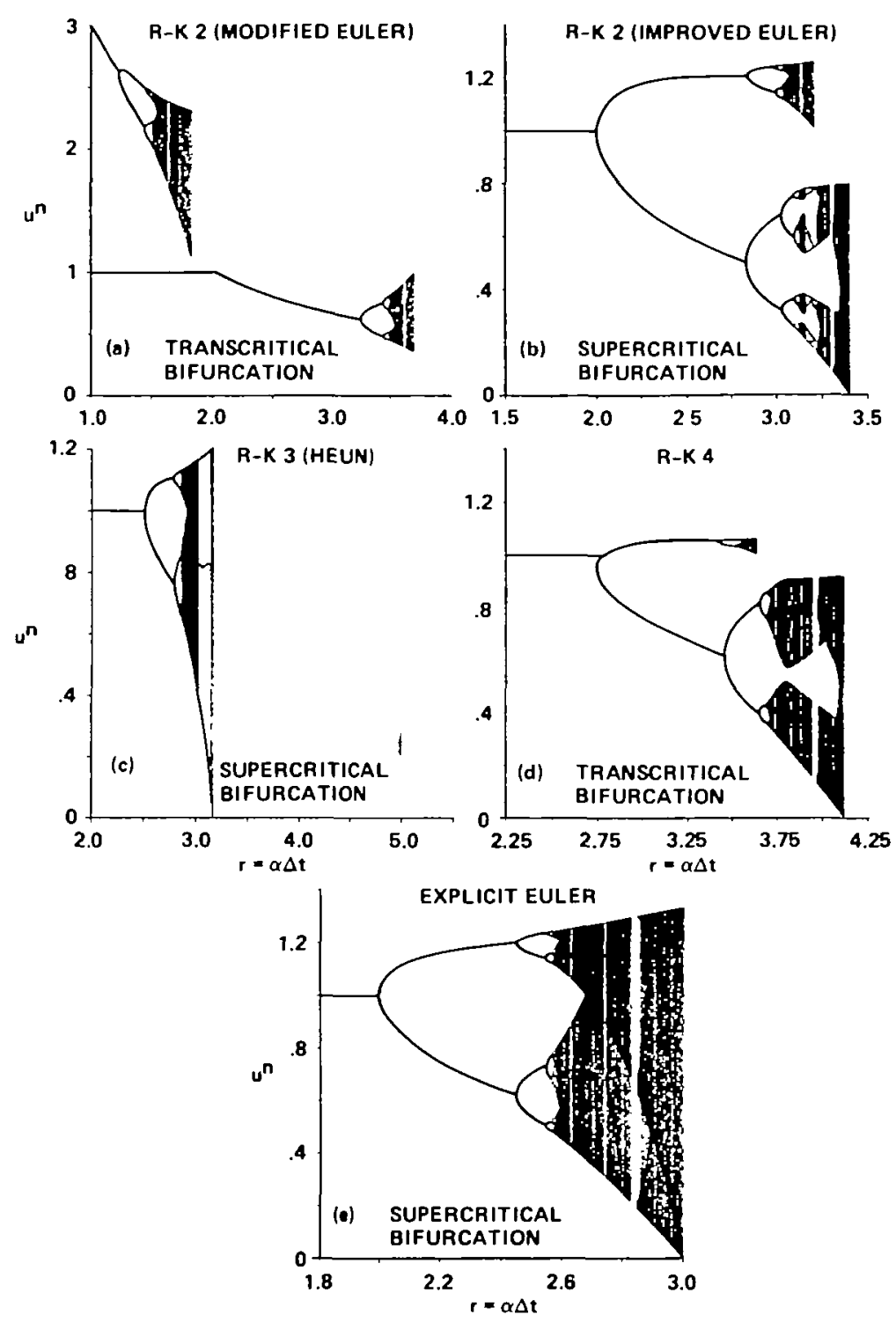

FIG. 2. Bifurcation diagrams for $u^{\prime}=\alpha u(1-u)$.

while the subscript $S$ indicates a spurious fixed point introduced by the numerical method.

The terms transcritical, supercritical and flip (period doubling) bifurcation [18] refer to the nature of the bifurcation of the fixed point at its linearised stability limit. Typically for one-dimensional problems both branches of supercritical bifurcations are stable while for transcritical bifurcations one branch is stable while the other (at least initially) is unstable. As can be seen, transcritical 

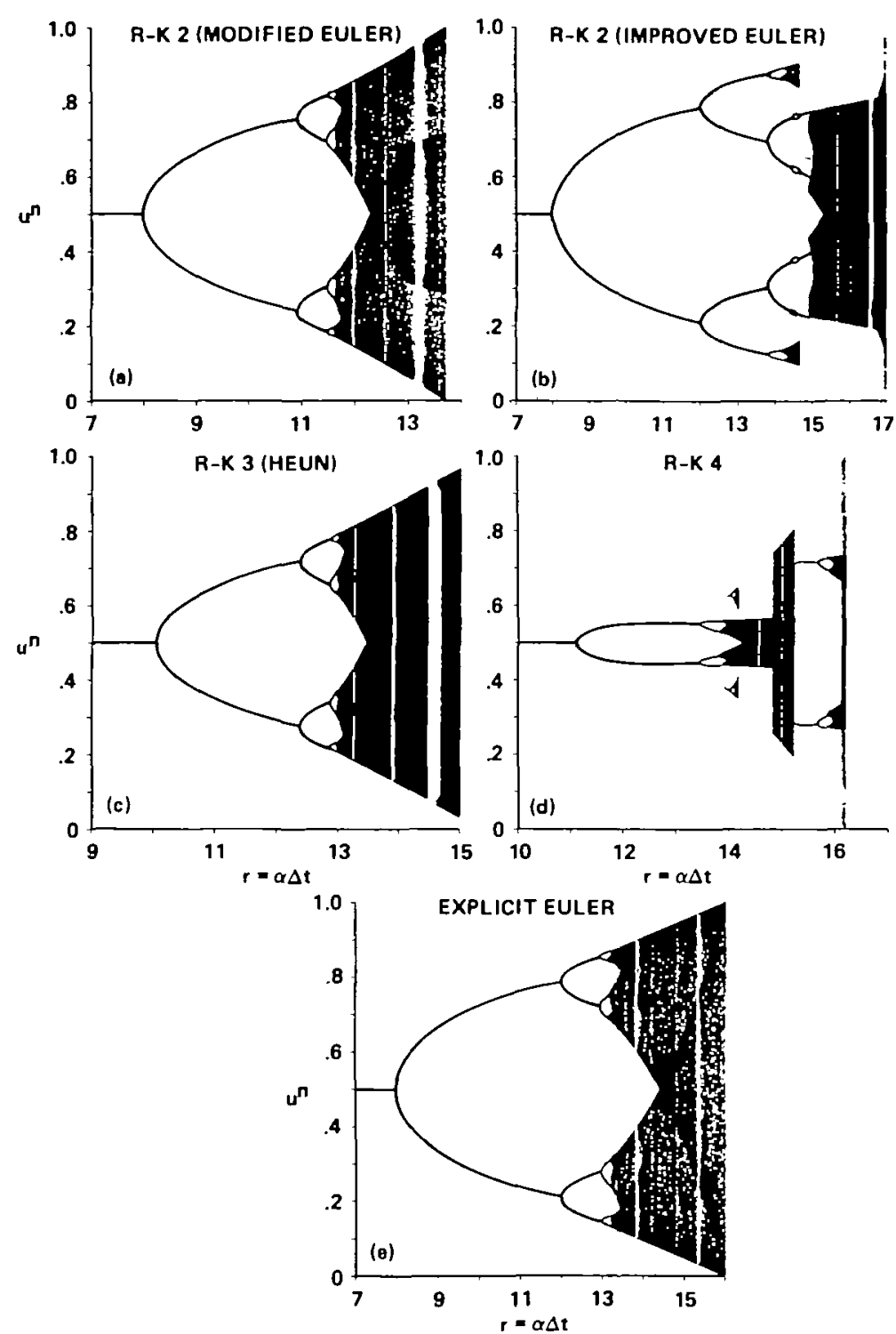

FIG. 3. Bifurcation diagrams (supercritical) for $u^{\prime}=\alpha u(1-u)\left(\frac{1}{2}-u\right)$.

bifurcations are invariably associated with situations in which there are stable spurious fixed points below the linearised stability limit of the scheme.

It is observed that, except for the explicit Euler scheme and the Heun scheme, there are spurious stable fixed points as well as the correct stable fixed point $\left(u^{*}=1\right.$ for $(1.1)$ and $u^{*}=\frac{1}{2}$ for the symmetric case of (1.4)). Although in the majority of cases these occur for values of $r$ above the linearised stability limit this is not always the case. In particular, for the modified Euler scheme applied to (1.1), we see from Fig. 2 that there is a stable spurious orbit that exists for all $\Delta t$ 
sufficiently small. The fourth order Runge-Kutta scheme applied to the same equation exhibits a spurious fixed point which is stable a little below the linearised stability limit (it could, for a different choice of nonlinearity, be stable substantially below this limit). It is clear that, when solving more complicated problems, such spurious points could be computed and mistaker. for the correct equilibrium.

Another interesting feature of the Figures is that, for certain methods, the true fixed point of the ODE may undergo a supercritical pitchfork bifurcation to a pair of branches, each of which represents spurious fixed points rather than the period doubling bifurcations that are familiar from the many accounts of the explicit Euler method (Fig. 1). This is illustrated in the bifurcation diagram for Improved Euler in Fig. 2. Such a bifurcation can recur as shown in the corresponding diagram in Fig. 3.

An important point to bear in mind when computing bifurcation diagrams is that several distinct stable periodic orbits may coexist for the same ranges of $r$ values. Any choice of initial value $u^{0}$ will, at best, be attracted to only one of these and consequently several different initial values must be used for each $r$ to ensure that a complete bifurcation diagram is produced. This is illustrated in several experiments described in an earlier report [15] on which this paper is based. Some solutions may be difficult to detect by numerical experimentation with bifurcation plots since their domains of attraction may be very small, although other numerical techniques may be used to solve (2.9) more directly.

In the next section we make use of perturbation arguments to investigate the local nature of the bifurcations from an essential fixed point of the differential equation to spurious period 1 rest states of explicit Runge-Kutta methods of order $\leqslant 4$.

\section{Branches of spurious fixed points}

We begin this section by investigating the behaviour of explicit $s$-stage Runge-Kutta methods in the neighbourhood of a stable fixed point $u^{*}$ of (2.1). The conditions under which spurious solutions bifurcate from the steady solution are established by Iserles et al. [6]. We extend these results by determining the nature of the bifurcation, its dependence on both the underlying differential equation and the specific Runge-Kutta method as well as investigating the stability of the bifurcated solution. Methods are said to be regular if they do not admit spurious fixed points $[4,5]$. Using the recursive test developed by Hairer et al. [4], it is readily verified that, of all the methods discussed in this paper, only the explicit Euler scheme is regular. For a general discussion of bifurcations of maps of the interval we refer to Whitley [18]. We shall subsequently address the issue of spurious fixed points that do not emanate from such bifurcations. Finally we shall make some remarks concerning the detection of spurious solutions.

It is convenient to express the general $s$-stage method in the form

$$
u^{n+1}=u^{n}+\Delta t \sum_{j=1}^{s} c_{j} f\left(z_{j}\right)
$$


where

$$
z_{j}=u^{n}+\Delta t \sum_{i=1}^{j-1} b_{j, l} f\left(z_{l}\right), \quad j=1,2, \ldots, s
$$

and, in the more standard notation of the previous section, $k_{j}=f\left(z_{j}\right)$. For the present we shall assume only that the method is consistent so that

$$
c^{T} e=1 \text {, }
$$

where $c=\left(c_{1}, c_{2}, \ldots, c_{s}\right)^{T}$ and $e=(1,1, \ldots, 1)^{T}$.

Clearly $u^{n}=u^{*}$ is a fixed point of (3.1), (3.2) if $u^{*}$ is such that $f\left(u^{*}\right)=0$. To investigate the stability of this fixed point we express the mapping (3.1), (3.2) in the form (2.8) and linearise about $u^{*}$ as described in the previous section. This leads to the stability function defined by $(2.11)$ and is given by

$$
R\left(u^{*}, \Delta t\right)=1+\rho c^{T}(I-\rho B)^{-1} e
$$

where $\rho=\Delta t f^{\prime}\left(u^{*}\right)$ and $B$ denotes the $s \times s$ array of weights $b_{j, l}$ that occur in (3.2). For explicit Runge-Kutta processes, the matrix $B$ is strictly lower triangular and, consequently, nilpotent. It then follows from the Neumann expansion that

$$
(I-\rho B)^{-1}=I+\rho B+\rho^{2} B^{2}+\cdots+\rho^{s-1} B^{s-1}
$$

and the right hand side of (3.4) is a polynomial of degree $s$ in $\rho$. For implicit Runge-Kutta methods (3.4) leads to a rational expression in $\rho$.

When the fixed point of the differential equation (2.1) is stable we must have $f^{\prime}\left(u^{*}\right)<0$ and the linearised stability condition (2.12) will then be satisfied for all $\Delta t$ sufficiently small since, from (3.4) and (3.3), it follows that

$$
R\left(u^{*}, \Delta t\right)=1+\Delta t f^{\prime}\left(u^{*}\right)+O\left(\Delta t^{2}\right) \text {. }
$$

Hence, $u^{*}$ will also be a stable fixed point of the Runge-Kutta method (3.1), (3.2) in some interval $\Delta t \in\left(0, \Delta t^{*}\right)$ or, equivalently, $\rho \in\left(\rho^{*}, 0\right)\left(\rho^{*} \equiv \rho^{*}(s)=\right.$ $\left.\Delta t^{*} f^{\prime}\left(u^{*}\right)<0\right)$. The interval $\left(\rho^{*}, 0\right)$ is usually called the interval of absolute stability of the Runge-Kutta method defined by (3.1), (3.2).

Because all the quantities involved are real a loss of stability can only be encountered when $\rho^{*}$ satisfies either

$$
\rho^{*} c^{T}\left(I-\rho^{*} B\right)^{-1} e=-2
$$

or

$$
\rho^{*} c^{T}\left(I-\rho^{*} B\right)^{-1} e=0 .
$$

In the former case it is seen from equation (2.10) that the perturbations from the fixed point satisfy $\delta^{n+1} \approx-\delta^{n}$, as $\rho$ decreases beyond $\rho^{*}$, leading to a period doubling (flip) bifurcation, while, in the latter case, $\delta^{n+1}$ and $\delta^{n}$ have the same sign and $\left|\delta^{n+1}\right|>\left|\delta^{n}\right|$. We shall focus on the second case here, leaving the period doubling situation to the next section. In both cases there is a loss of stability of the fixed point $u^{*}$ for $\rho<\rho^{*}$ that would, for a linear problem, lead to $\left|u^{n}\right| \rightarrow \infty$ as $n \rightarrow \infty$. One of our aims here is to show that such divergence need not occur for a 
genuinely nonlinear differential equation $\left(f^{\prime}(u) \neq\right.$ constant) but that there may be a bifurcation to a fixed point, $u_{\Delta}^{*}$, that is spurious in the sense that $f\left(u_{\Delta}^{*}\right) \neq 0$ which excludes it from being a stationary point of (2.1). We also indicate how the nature of the bifurcation is influenced by properties of the method and those of $f(u)$.

Any fixed point, $u_{\Delta}^{*}$, of (3.1), (3.2) must satisfy

$$
\sum_{j=1}^{s} c_{j} f\left(z_{j}\right)=0
$$

and

$$
z_{j}=u_{\Delta}^{*}+\Delta t \sum_{l=1}^{j-1} b_{j, l} f\left(z_{l}\right), \quad j=1,2, \ldots, s .
$$

Defining the small quantity

$$
\varepsilon=u_{\Delta}^{*}-u^{*},
$$

we seek a solution of (3.7) and (3.8) for $\rho$ close to $\rho^{*}\left(\Delta t\right.$ close to $\left.\Delta t^{*}\right)$ in the form of perturbation expansions given by

$$
\Delta t=\Delta t^{*}+a \varepsilon+b \varepsilon^{2}+\cdots
$$

and

$$
z_{j}=u^{*}+\alpha_{j} \varepsilon+\beta, \varepsilon^{2}+\gamma_{j} \varepsilon^{3}+\ldots, j=1,2, \ldots, s .
$$

From this last expansion we deduce that

$$
\begin{aligned}
f\left(z_{j}\right)= & \varepsilon f^{\prime}\left(u^{*}\right) \alpha_{j}+\frac{1}{2} \varepsilon^{2}\left[f^{\prime \prime}\left(u^{*}\right) \alpha_{j}^{2}+2 f^{\prime}\left(u^{*}\right) \beta_{j}\right] \\
& +\varepsilon^{3}\left[\frac{1}{6} f^{m}\left(u^{*}\right) \alpha_{j}^{3}+f^{\prime \prime}\left(u^{*}\right) \alpha_{j} \beta_{j}+f^{\prime}\left(u^{*}\right) \gamma_{j}\right]+\ldots
\end{aligned}
$$

Substituting (3.10)-(3.12) into (3.8) and equating like powers of $\varepsilon$ leads to

$$
\left(I-\rho^{*} B\right) \alpha=e,
$$

and

$$
\left(I-\rho^{*} B\right) \boldsymbol{\beta}=B\left[a f^{\prime}\left(u^{*}\right) \boldsymbol{\alpha}+\frac{1}{2} \Delta t^{*} f^{\prime \prime}\left(u^{*}\right) \boldsymbol{\alpha}^{2}\right]
$$

where $a$ is to be determined, $\alpha=\left(\alpha_{1}, \ldots, \alpha_{s}\right)^{T}$ and $\alpha^{2}$ denotes the vector whose components are the squares of the corresponding elements of $\alpha$. It is convenient for subsequent manipulations to rearrange (3.14) to read

$$
\rho^{*}\left(I-\rho^{*} B\right) \boldsymbol{\beta}=\left[I-\left(I-\rho^{*} B\right)\right]\left[a f^{\prime}\left(u^{*}\right) \boldsymbol{\alpha}+\frac{1}{2} \Delta t^{*} f^{\prime \prime}\left(u^{*}\right) \boldsymbol{\alpha}^{2}\right]
$$

Since (3.6) and (3.13) imply that $c^{T} \boldsymbol{\alpha}=0$ we find, on combining (3.7) and (3.12) and neglecting terms in $\varepsilon^{3}$, that the condition for a fixed point becomes

$$
f^{\prime \prime}\left(u^{*}\right) c^{T} \alpha^{2}+2 f^{\prime}\left(u^{*}\right) c^{T} \beta=0
$$

or, with $\rho^{*}=f^{\prime}\left(u^{*}\right) \Delta t^{*}$,

$$
\Delta t^{*} f^{\prime \prime}\left(u^{*}\right) c^{T} a^{2}+2 \rho^{*} c^{T} \beta=0
$$


Taking this together with (3.15) we obtain

$$
a=-\frac{\Delta t^{*} f^{\prime \prime}\left(u^{*}\right)}{2 f^{\prime}\left(u^{*}\right)} \frac{c^{T}\left(I-\rho^{*} B\right)^{-1} \alpha^{2}}{c^{T}\left(I-\rho^{*} B\right)^{-1} \alpha}
$$

where $\alpha$ is given by the solution of the system (3.13). Thus $a$ is well-defined provided that $c^{T}\left(I-\rho^{*} B\right)^{-1} \alpha$ does not vanish.

When $a \neq 0$, that is, when

$$
f^{\prime \prime}\left(u^{*}\right) \neq 0
$$

and

$$
c^{T}\left(I-\rho^{*} B\right)^{-1} \alpha^{2} \neq 0
$$

there is a transcritical bifurcation given, to first order, by (3.9) and (3.10). The first of these conditions depends on the differential equation (and is violated, for example, when $f(u)=\alpha u(1-u)\left(\frac{1}{2}-u\right)$ with $\left.u^{*}=\frac{1}{2}\right)$ and the second condition depends solely on the Runge-Kutta method.

To study the stability of the bifurcating solution we write

$$
\lambda(\varepsilon)=R\left(u_{\Delta}^{*}, \Delta t\right)
$$

to denote the Jacobian of the mapping at $u_{\Delta}^{*}=u^{*}+\varepsilon, \Delta t=\Delta t^{*}+a \varepsilon+\ldots$ We then find that

$$
\lambda^{\prime}(0)=\frac{1}{2} \Delta t^{*} f^{\prime \prime}\left(u^{*}\right) c^{T}\left(I-\rho^{*} B\right)^{-1} \boldsymbol{a}^{2}
$$

and, since $\lambda(0)=1$, we shall have $\lambda(\varepsilon)<1$ provided

$$
\varepsilon f^{\prime \prime}\left(u^{*}\right) c^{T}\left(I-\rho^{*} B\right)^{-1} \alpha^{2}<0 .
$$

This condition determines the sign of $\varepsilon$ that gives rise to a stable branch. Using (3.13), it may be shown that

$$
c^{T}\left(I-\rho^{*} B\right)^{-1} a=\left.\frac{d}{d \rho}\left(1+\rho c^{T}(I-\rho B)^{-1} e\right)\right|_{\rho=\rho^{*}}
$$

Moreover, as we are considering specifically the case when (3.6) holds at the bifurcation, the argument on the right must be a decreasing function of $\rho$ at $\rho^{*}$. Thus, by the definition of interval of absolute stability, it follows that $c^{T}(I-$ $\left.\rho^{*} B\right)^{-1} a<0$. This result together with (3.16) imply that the stable branch emanating from $\left(u^{*}, \Delta t^{*}\right)$ is given by (3.9) and (3.10) for $a \varepsilon>0$, that is, when $\Delta t>\Delta t^{*}$. The consequences of this result are twofold. Not only does it imply that a spurious rest state may be reached for $\Delta t>\Delta t^{*}$ but also, that an unstable branch exists for $\Delta t<\Delta t^{*}$ which may substantially affect the domain of attraction of the true, stable rest state.

In those cases where one or other of conditions (3.17), (3.18) is not satisfied we find that $a=0$ in (3.10) and higher order expansions are necessary to determine the nature of the bifurcation. Omitting the details, the coefficient of $\varepsilon^{2}$ in (3.10) is found to be

$$
b=-\frac{\rho^{*}}{6 f^{\prime}\left(u^{*}\right)^{3}} \frac{c^{T}\left(I-\rho^{*} B\right)^{-1} \alpha^{*}}{c^{T}\left(I-\rho^{*} B\right)^{-1} \alpha}
$$


where

$$
\boldsymbol{\alpha}^{*}=\operatorname{diag}\left(\boldsymbol{\alpha}^{*}\right)\left[\left(f^{\prime}\left(u^{*}\right) f^{\prime \prime}\left(u^{*}\right)-3 f^{\prime \prime}\left(u^{*}\right)^{2}\right) \boldsymbol{\alpha}^{2}+3 f^{\prime \prime}\left(u^{*}\right)^{2}\left(I-\rho^{*} B\right)^{-1} \boldsymbol{\alpha}^{2}\right] .
$$

When $a=0$ and $b \neq 0$ the bifurcation at $\Delta t=\Delta t^{*}$ will be of pitchfork type (see Whitley [18]). Moreover, it will be supercritical if $b>0$ and subcritical if $b<0$ with the former being stable and the latter unstable.

Thus, where loss of linear stability occurs at $\rho^{*}$ defined by (3.6), the solution of the corresponding Runge-Kutta method bifurcates to a spurious steady state given locally by the parameterisation (3.9) and (3.10). When the coefficient $a$, given by (3.16), is non-zero the bifurcation is of transcritical type while, if $a=0$ but the expression (3.20) for $b$ is non-vanishing, the bifurcation is of pitchfork type. By including higher order terms in (3.10) the nature of higher degree bifurcations may be determined in a similar manner.

We now restrict attention to explicit Runge-Kutta methods that have order $s$ with $s$ stages. This requires that $s \leqslant 4[7]$ and the linear stability function of such methods is unique for each $s$ and given by

$$
R\left(u^{*}, \Delta t\right)=1+\rho+\frac{1}{2 !} \rho^{2}+\cdots+\frac{1}{s !} \rho^{s},
$$

an $O\left(\rho^{s+1}\right)$ approximation to $e^{\rho}$. Thus, all $s$ th order methods with $s$ stages have the same linearised stability limit. Moreover, it follows that

$$
c^{T}(I-\rho B)^{-1} e=1+\frac{1}{2 !} \rho+\cdots+\frac{1}{s !} \rho^{s-1}
$$

from which it is easily shown that $\rho^{*}$ satisfies (3.5) for $s=1$ (Euler) and $s=3$ (Heun), leading to a flip bifurcation, whereas (3.6) is satisfied for $s=2$ (modified and Improved Euler) and $s=4(R-K 4)$. It is for these last two cases that the theory developed in this section applies.

For $s=2$, the most general second order, two stage Runge-Kutta method may be parameterised by $\theta(\neq 0)$ with $c=(1-\theta, \theta)^{T}$ and

$$
B=\left(\begin{array}{cc}
0 & 0 \\
1 /(2 \theta) & 0
\end{array}\right) \text {. }
$$

It is easily deduced that $\rho^{*}=-2, c^{T}\left(I-\rho^{*} B\right)^{-1} a=-1$ for all $\theta$ and

$$
c^{T}\left(I-\rho^{*} B\right)^{-1} a^{2}=\frac{1-2 \theta}{\theta} \text {. }
$$

Thus, the method will generate a transcritical bifurcation except when $\theta=\frac{1}{2}$ (the improved Euler method (2.5)). For $\theta \neq \frac{1}{2}$ we obtain

$$
a=\frac{\Delta t^{*} f^{\prime \prime}\left(u^{*}\right)}{f^{\prime}\left(u^{*}\right)} \frac{1-2 \theta}{\theta}
$$

In particular, for the modified Euler method $(\theta=1)$ with $f(u)=\alpha u(1-u)$ and $u^{*}=1$ we have $a=-\Delta t^{*}$. Thus, from (2.13), (3.9) and (3.10), the bifurcation 
occurs at $r(=\alpha \Delta t)=2$ and is described to first order by

$$
r \approx 2\left(2-u_{\Delta}^{*}\right)
$$

and, for stability, $r>2$ so that $u_{\Delta}^{*}<1$. These results are seen to agree with the graphical results shown in Fig. 2.

For the fourth order Runge-Kutta method, the limit of absolute stability is $\rho^{*} \approx-2.785$ as given by the negative real root of (3.5) with $s=4$. Following a tedious calculation we find that (3.16) gives

$$
a \approx-0.9598 f^{\prime \prime}\left(u^{*}\right) /\left[f^{\prime}\left(u^{*}\right)\right]^{2}
$$

so that a transcritical bifurcation always occurs provided $f^{\prime \prime}\left(u^{*}\right) \neq 0$. The equation of the tangent line at the bifurcation point is given by

$$
u_{\Delta}^{*} \approx 1+0.521(r-2 \cdot 785)
$$

for $f(u)=\alpha u(1-u)$ with $r>2.785$ so that $u_{\Delta}^{*}>1$ for stability. These findings agree with the graphical results shown in Fig. 2 .

Of the examples we have looked at in this section, only the improved Euler scheme leads to a pitchfork bifurcation through violation of condition (3.18). In this case we find that

$$
b=\frac{f^{\prime}\left(u^{*}\right) f^{\prime \prime \prime}\left(u^{*}\right)-3 f^{\prime \prime}\left(u^{*}\right)^{2}}{3 f^{\prime}\left(u^{*}\right)^{3}}
$$

The other instances of pitchfork bifurcations occur through failure of (3.17) and the expression for $b$ simplifies to

$$
b=-\frac{\rho^{*} f^{\prime \prime \prime}\left(u^{*}\right)}{6 f^{\prime}\left(u^{*}\right)^{2}} \frac{c^{T}\left(I-\rho^{*} B\right)^{-1} a^{3}}{c^{T}\left(I-\rho^{*} B\right)^{-1} a} .
$$

This leads to

$$
b=\frac{f^{\prime \prime}\left(u^{*}\right)}{f^{\prime}\left(u^{*}\right)^{2}} \frac{1-3 \theta+3 \theta^{2}}{3 \theta^{2}}
$$

for the general second order Runge-Kutta method and

$$
b=2 \cdot 187 \frac{f^{\prime \prime \prime}\left(u^{*}\right)}{f^{\prime}\left(u^{*}\right)^{2}}
$$

for RK4. The factor involving $\theta$ in (3.22) is always positive and it is interesting to note that the value obtained for $b$ is the same for both $\theta=1$ (modified Euler) and $\theta=\frac{1}{2}$ (improved Euler). This is in accordance with the results shown in Fig. 3 (at $\Delta t=9$, for example). Thus, a (stable) supercritical pitchfork bifurcation results in all cases provided that $f^{\prime \prime}\left(u^{*}\right)>0$. For instance, the function $f(u)=u(1-u)\left(\frac{1}{2}-\right.$ $u)=-\frac{1}{4}\left(u-\frac{1}{2}\right)+\left(u-\frac{1}{2}\right)^{3}$ has three real zeros and, with $u^{*}=\frac{1}{2}, f^{m}\left(u^{*}\right)=6$. On the other hand, for the function $f(u)=-\frac{1}{4}\left(u-\frac{1}{2}\right)-\left(u-\frac{1}{2}\right)^{3}$ which has only one real zero, $u^{*}=\frac{1}{2}, f^{\prime \prime}\left(u^{*}\right)=-6$ and an (unstable) subcritical pitchfork bifurcation would result. 
The perturbation analysis described in this section has shown that it is possible to predict not only the onset of instability at an essential stationary point $u^{*}$ of the differential system but also to determine the nature of bifurcation that occurs and the stability along the bifurcating branch.

It was remarked in the previous section that information regarding branches of spurious solutions could be determined by considering the behaviour at large values of $\Delta t$. We now address this issue in a little more detail. We shall suppose that $f(u)$ is a polynomial of degree $m$ having $\mu(\leqslant m)$ real zeros. Then, if the Runge-Kutta matrix $B$ has rank $s-1$ and the vector $c$ has $c_{s} \neq 0$ (to ensure that the $s$ 'th iterate of $f$ is used in (3.1)), equation (2.9) becomes

$$
f\left(u_{\Delta}^{*}\right)^{m^{s-1}}=0
$$

for $\Delta t \rightarrow \infty$. Thus, for large $\Delta t$, each of the fixed points of (2.1) generates a fixed point of multiplicity $m^{s-1} \cdot m^{s-1}-1$ of these are spurious and may then be developed in asymptotic expansions in inverse powers of $\Delta t(r)$ thereby providing approximations to sections of the branches of spurious solutions in the $\left(u_{\Delta}^{*}, r\right)$ plane. We shall illustrate the argument for Heun's method applied to (1.1) though it may be readily extended. In this case $m=2$ and $s=3$ so that there are six spurious roots at $r=\infty$. These have the asymptotic expansions

$$
\begin{gathered}
u_{\Delta}^{*}=\frac{9}{2 r^{2}}-\frac{27}{4 r^{3}}+\ldots, \\
u_{\Delta}^{*}=\frac{3}{r}-\frac{9}{2 r^{2}}+\ldots \\
u_{\Delta}^{*}=\frac{3}{r}+\frac{18}{r^{3}}+\ldots
\end{gathered}
$$

for the triple spurious root near $u=0$ and

$$
\begin{gathered}
u_{\Delta}^{*}=1-\frac{9}{2 r^{2}}-\frac{27}{4 r^{3}}+\ldots, \\
u_{\Delta}^{*}=1+\frac{3}{r}+\frac{9}{2 r^{2}}+\ldots, \\
u_{\Delta}^{*}=1+\frac{3}{r}+\frac{18}{r^{3}}+\ldots,
\end{gathered}
$$

for the triple spurious root near $u=1$. For large $r(\Delta t)$ the roots $(3.24 \mathrm{a}-\mathrm{c})$ and (3.25a) lie in the interval $(0,1)$ while those of $(3.25 \mathrm{~b}-\mathrm{c})$ lie in $(1, \infty)$. Furthermore, since equation (3.6) has no real roots $\rho^{*}$ for Heun's method when either $u^{*}=0,1$, the branches of the solutions cannot cross either of these two lines in the $\left(u_{\Delta}^{*}, r\right)$ plane. As $r$ decreases to zero, the branches given by $(3.25 b-c)$ must either tend to infinity or must coalesce (as the two roots merge to form a complex conjugate pair). It may be shown that the latter case occurs and the two roots coalesce at $u_{\Delta}^{*}=3 / 2, r \approx 6.749$ giving rise to a saddle-node bifurcation [17]. 
The remaining four roots $(3.24 a-c)$ and (3.25a) must be strictly contained in the semi-infinite strip $(0<u<1, r>0)$. There are no spurious roots for small positive values of $r$ so there must be two separate branches one connecting $(u=0, r=\infty)$ to itself and the other connecting $(u=0, r=\infty)$ to $(u=1, r=\infty)$. In fact (3.24a) and (3.24b) merge at $r \approx 4.915\left(u_{\Delta}^{*} \approx 0.25\right)$ while (3.24c) and (3.25a) merge at $r \approx 6.480\left(u_{\Delta}^{*} \approx 0.695\right)$ thus leading to two further saddle-node bifurcations.

At a saddle-node bifurcation the function $\Phi(u, \Delta t)$ has a double root, $u=u_{\Delta}^{*}$, and consequently $\Phi_{u}\left(u_{\Delta}^{*}, \Delta t\right)=0$. We may assume that $\Phi_{u u}\left(u_{\Delta}^{*}, \Delta t\right) \neq 0$ for Heun's method, for otherwise $\Phi$ would have a zero of multiplicity three at this point. (This situation would occur if these branches were themselves to have been generated from a supercritical bifurcation of period 1 . See, for example, both $R-K 2$ methods in Fig. 3.) Thus, $\Phi_{u}(u, \Delta t)$ must change sign as the branch of solutions is crossed and, by (2.11-2.12), one of the branches must correspond to a stable spurious solution in the neighbourhood of a saddle-node bifurcation and the other to an unstable branch. In the present example it is straightforward to show that it is the upper branch that is stable for $u>1$ and the lower of the two branches for $0<u<1$ (one of these is apparent in the bifurcation diagram for $R-K 3$ (Heun) in Fig. 2. See also Table 2.1). The intervals of stability of these solutions are given (approximately) in Table 2.1 and are seen to be extremely small.

Given that spurious solutions may be generated by Runge-Kutta methods of the type (3.1), (3.2), it is appropriate to ask whether these can be detected when solving systems for which the true fixed points are not known. One way in which this can be done is to compute a secondary estimate for $u^{n+1}$ using an expression of the type (3.1) but using a different weighting vector, $\hat{c}$, say. Then discrepancies between the primary and secondary estimates that exceeded the expected level of truncation error would point to the existence of spurious equilibria. (Note that the two estimates would coincide not only at a true fixed point but also in the unlikely event that the underlying methods possessed a common spurious fixed point.) Embedded Runge-Kutta methods of this kind are commonly used in variable time-stepping codes $[3,12]$ and their use is perhaps the surest safeguard against computing erroneous fixed points.

In the next section we investigate some of the higher order orbits of the schemes where consecutive iterates of (2.8) oscillate between two or more values (a feature not present in differential equations). It was shown earlier in this section that, for $s$ th order, $s$-stage methods, such bifurcations occur for odd values of $s$ and at values of $\Delta t$ dictated by (3.5). The orbits in these cases are generally much more difficult to obtain analytically, even with the aid of algebraic manipulation software, and only a cursory analysis is presented.

\section{Orbits of period two}

If we use a difference scheme to solve an ODE using a value of $r$ which is slightly above the stability limit for a fixed point of the scheme (either spurious or one belonging to the differential equation) then we often find that the iteration (2.8) 
will oscillate between two values. This is known as period doubling, a process which is often repeated again at the stability limit for the period two orbit and so on. It is this process which is illustrated by the bifurcation diagrams. As well as cascades of period doubling bifurcations, there are generally periods of all orders as well as chaotic regions.

We consider next the case of period two orbits. This means that there must exist two values $u^{\circ}$ and $u^{\bullet}$ such that

$$
\begin{aligned}
& u^{\circ}=u^{\bullet}+\Delta t \Phi\left(u^{\bullet}, \Delta t\right) \\
& u^{\bullet}=u^{\circ}+\Delta t \Phi\left(u^{\circ}, \Delta t\right),
\end{aligned}
$$

that is,

$$
\Phi\left(u^{\circ}, \Delta t\right)+\Phi\left(u^{\bullet}, \Delta t\right)=0 .
$$

Rewriting this in terms of just one of the values, $u^{\bullet}$ say, we have that period two orbit states are given by the solutions of the equation

$$
\Phi\left(u^{\bullet}, \Delta t\right)+\Phi\left(u^{\bullet}+\Delta t \Phi\left(u^{\bullet}, \Delta t\right), \Delta t\right)=0 .
$$

There will be at least two solutions of (4.3). If there are more they must be paired through (4.2). Comparing with (2.9), it is seen that (4.2) and (4.3) will also be satisfied by the fixed points of the scheme $u^{\bullet}=u^{\circ}=u_{\Delta}^{*}$. Applying a similar argument to that used earlier in this paper, if $f$ is a polynomial of degree $m$ and the Runge-Kutta method has $s$ stages, $\Phi(u, \Delta t)$ is a polynomial of degree $m^{s}$ in $u$. However, for period two orbits, $\Phi$ is iterated in equation (4.3) and is consequently a polynomial of degree $m^{2 s}$. Thus a situation of bewildering complexity can be achieved with very modest values of $m$ and $s$.

As a prelude to investigating stability, the period 2 orbit $\left(u^{\bullet}, u^{\circ}, u^{\bullet}, \ldots\right)$ is perturbed by writing

$$
u^{2 n}=u^{\bullet}+\varepsilon v^{2 n}, \quad u^{2 n+1}=u^{0}+e v^{2 n+1}
$$

(see e.g. Sleeman et al. [14] for a similar technique applied to linear multistep schemes). Substituting these into (2.8) and neglecting second order terms in $\varepsilon$ leads to

$$
v^{2 n+1}=R\left(u^{\bullet}, \Delta t\right) v^{2 n}, \quad v^{2 n+2}=R\left(u^{\circ}, \Delta t\right) v^{2 n+1}
$$

where $R$ is defined by (2.11). Thus, $v^{n} \rightarrow 0$ as $n \rightarrow \infty$, as required for a stable orbit, if and only if

$$
\left|R\left(u^{\bullet}, \Delta t\right)\right|\left|R\left(u^{\circ}, \Delta t\right)\right|<1 \text {. }
$$

The algebraic details become too involved for all but the simplest methods and non-linearities and we therefore present the analytic forms for the period two orbits only for the explicit Euler scheme. These are shown in Table 4.1. The many stable orbits of period two that exist for the other Runge-Kutta methods are displayed in Fig. 2 and 3.

Comparing equation (2.4) and (4.3) (with $\Phi(u, \Delta t)=f(u)$ ) it is seen that the equations that define a period 2 orbit of the explicit Euler scheme are precisely those that define the fixed points of the improved Euler scheme. The stable 
TABLE 4.1

Period 2 orbits of the explicit Euler scheme

\begin{tabular}{ccl}
\hline Equation & Period 2 orbits & \multicolumn{1}{c}{ Stable range } \\
\hline$u^{\prime}=\alpha u(1-u)$ & $\frac{r+2 \pm \sqrt{r^{2}-4}}{2 r}$ & $2<r<\sqrt{6} \approx 2.4495$ \\
$u^{\prime}=\alpha u(1-u)\left(\frac{1}{2}-u\right)$ & $\frac{1 \pm \sqrt{1-8 / r}}{2}$ & $8<r<12$ \\
& $\frac{1}{2}-\frac{\sqrt{1-12 / r}}{4} \pm \frac{\sqrt{1+4 / r}}{4}$ & $12<r<14$ \\
& $\frac{1}{2}+\frac{\sqrt{1-12 / r}}{4} \pm \frac{\sqrt{1+4 / r}}{4}$ & $12<r<14$ \\
\hline
\end{tabular}

ranges in the two situations are, however, different. We also observe in Table 4.1 that several stable periodic orbits may coexist over a range of $r$ values.

The fact that a numerical method is attracted to an orbit of period 2 is much less important than its attraction to a spurious fixed point since the former is readily identified provided that the solution is observed over at least two consecutive time steps (it would also be detected by using an embedded $R-K$ method). Perhaps the most serious implication of a (stable or unstable) period 2 orbit is when it occurs below the linearised stability limit where it may have a deleterious effect on the domain of attraction of the true fixed point.

\section{Summary}

We have investigated the fixed points and periodic orbits of four Runge-Kutta schemes, contrasting them with those of the explicit Euler scheme which is known to possess only the fixed points of the differential equation. We have seen that, not only do these schemes produce spurious fixed points, but that these spurious features of the schemes can manifest themselves below the linearised stability limit for the correct fixed points. This raises the possibility of erroneous results being produced when such schemes are used for computations on problems where the correct result is not known a priori. We have also observed how multiple orbits of a given period may co-exist, the particular one selected by the scheme being dependent on the initial data.

Future work will be directed towards investigations into the effect of using such ODE solvers for the source term component of reaction-convection equations and other partial differential equations.

\section{REFERENCES}

1. BrezzI, F., Ushikı, S., \& FuJII, H. 1984 Real and ghost bifurcation dynamics in difference schemes for ODEs, in Numerical Methods for Bifurcation Problems (T. Kupper, H. D. Mittelman, H. Weber eds.), Basel: Birkhauser-Verlag, 79-104.

2. DerIve, algebraic manipulation package for IBM PC compatibles, Austria: Uniware. 
3. Hairer, E., Norsett, S. P., \& Wanner, G. 1987 Solving Ordinary Differential Equations I. Nonstiff Problems, Berlin: Springer-Verlag.

4. Hairer, E., Iserles, A., \& Sanz-Serna, J. M. 1990 Equilibria of Runge-Kutta methods, Numer. Math. 58, 243-254.

5. IsERLES, A. 1990 Stability and dynamics of numerical methods for nonlinear ordinary differential equations, IMA J. Numer. Anal., 10, pp 1-30.

6. Iserles, A., Peplow, A. T., \& Stuart, A. M. 1991 A unified approach to spurious solutions introduced by time discretisation Part I: Basic Theory, SIAM J. Numer. Anal. 28.

7. Lambert, J. D. 1973 Computational Methods in Ordinary Differential Equations, J. Wiley and Sons.

8. MAPLE, algebraic manipulation package, University of Waterloo, Canada.

9. MAY, R. T. 1976 Simple mathematical models with very complicated dynamics, Nature 261, 459-467.

10. Mrtchell, A. R., \& Griffiths, D. F. 1986 Beyond the stability limit in non-linear problems, Pitman Research Notes in Mathematics Series, 140 Numerical Analysis, (D. F. Griffiths and G. A. Watson, eds.), pp 140-156.

11. NewELl, A. C. 1977 Finite amplitude instabilities of partial difference equations, SIAM J. Appl. Math. 33, 133-160.

12. SAnz-Serna, J. M. 1990 Numerical ordinary differential equations vs. dynamical systems, Applied Maths. and Computation Repor 1990/3, University of Valladolid.

13. Sanz-Serna, J. M., \& GrifftThs, D. F. 1991 A new class of results for the algebraic equations of implicit Runge-Kutta processes, IMA J. Numer. Anal. 11, 449-455.

14. Sleeman, B. D., Griffiths, D. F., Mrtchell, A. R., \& Smith, P. D. 1988 Stable periodic solutions in nonlinear difference equations, SIAM J. Sci. Stat. Comput., 9, pp 543-557.

15. Sweby, P. K., Yee, H. C., \& Grifftrhs, D. F. 1990 On spurious steady-state solutions of explicit Runge-Kutta schemes, Numerical Analysis Report 3/90, University of Reading.

16. Thompson, J. M. T., \& Stewart, H. B. 1988 Nonlinear Dynamics and Chaos, J. Wiley and Sons.

17. Verhulst, F. 1990 Nonlinear Differential Equations and Dynamical Systems, Berlin: Springer-Verlag.

18. Whitley, D., 1983 Discrete dynamical systems in dimensions one and two, Bull. London Math. Soc. 15, pp 177-217.

19. YEE, H. C. 1989 A class of high resolution explicit and implicit shock capturing methods, NASA TM-101088.

20. Yee, H. C., Sweby, P. K., \& Griffrths, D. F. 1991. Dynamical approach study of spurious steady state numerical solution of nonlinear differential equations. I The ODE connection and its implications for algorithm developments in computational fluid dynamics, J. Comp. Phys., 97, 249-310. 